\title{
Backdoor Attack on Deep Neural Networks Triggered by Fault Injection Attack on Image Sensor Interface
}

\author{
Tatsuya Oyama \\ Ritsumeikan University \\ Kusatsu, Shiga, Japan \\ ri0068hi@ed.ristumei.ac.jp \\ Kota Yoshida \\ Ritsumeikan University \\ Kusatsu, Shiga, Japan \\ ri0044ep@ed.ristumei.ac.jp
}

\author{
Shunsuke Okura \\ Ritsumeikan University \\ Kusatsu, Shiga, Japan \\ sokura@fc.ritsumei.ac.jp \\ Takeshi Fujino \\ Ritsumeikan University \\ Kusatsu, Shiga, Japan \\ fujino@se.ristumei.ac.jp
}

\begin{abstract}
Recent automobiles use image sensors to take in the physical world information, and deep neural networks (DNNs) are used to recognize the surroundings to control the vehicles. Adversarial examples and backdoor attacks that induce misclassification by tampering with input images to DNN have been proposed as methods of attacking DNNs. As an example of attacks on DNNs equipped in automobiles, a method has been reported in which an adversarial mark is added to input images by physically putting a sticker on a road sign. However, the method reduces reproducibility due to the influence of the shooting environment. The tampering area needs to be increased to improve reproducibility. However, these large marks are easily seen by people.

We propose a method of adding an adversarial mark for triggering backdoor attacks by a fault injection attack on the Mobile Industry Processor Interface (MIPI), which is the popular CMOS image sensor interface. This method can increase the reproducibility of attacks. We attack the MIPI to add adversarial marks to the input image. Two attack drivers are electrically connected to the MIPI data lane in our attack system. Most image signals are transferred from the sensor to the processor by canceling the attack signal. Then, the adversarial mark is added by activating the attack signal. An adversary aiming to carry out backdoor attacks mixes poison data, which consists of images tampered with adversarial marks at specific locations and of adversarial target classes, into a training dataset. The backdoor model classifies images with adversarial marks into an adversarial target class and other images into the correct classes.

We conducted backdoor attack experiments with the MNIST dataset for handwritten digit recognition and the German Traffic Sign Recognition Benchmark (GTSRB) dataset for traffic sign recognition. Attacks on MNIST had a $91 \%$ success rate with $1 \%$ poison
\end{abstract}

Permission to make digital or hard copies of all or part of this work for personal or classroom use is granted without fee provided that copies are not made or distributed for profit or commercial advantage and that copies bear this notice and the full citation on the first page. Copyrights for components of this work owned by others than ACM must be honored. Abstracting with credit is permitted. To copy otherwise, or republish, to post on servers or to redistribute to lists, requires prior specific permission and/or a fee. Request permissions from permissions@acm.org.

ASHES '21, November 19, 2021, Virtual Event, Republic of Korea.

(c) 2021 Association for Computing Machinery.

ACM ISBN 978-1-4503-8662-3/21/11 . \$15.00

https://doi.org/10.1145/3474376.3487287 data, and attacks on GTSRB had a $92 \%$ success rate with $5.1 \%$ poison data.

\section{CCS CONCEPTS}

- Security and privacy $\rightarrow$ Hardware attacks and countermeasures.

\section{KEYWORDS}

backdoor attack, MIPI, image sensor interface, deep neural networks, fault injection attack

\section{ACM Reference Format:}

Tatsuya Oyama, Shunsuke Okura, Kota Yoshida, and Takeshi Fujino. 2021. Backdoor Attack on Deep Neural Networks Triggered by Fault Injection Attack on Image Sensor Interface. In Proceedings of the 5th Workshop on Attacks and Solutions in Hardware Security (ASHES '21), November 19, 2021, Virtual Event, Republic of Korea. ACM, New York, NY, USA, 10 pages. https: //doi.org/10.1145/3474376.3487287

\section{INTRODUCTION}

Recent automobiles are equipped with an advanced driver assistance system (ADAS), which controls systems such as accelerators, brakes, and steering wheels by recognizing surrounding vehicles and objects while driving. In addition to notifying the driver of traffic sign recognition results, a system for automatic driving that is aware of speed limits has been developed. In such a system, image sensors take in the physical world information, and the automobile is controlled by recognizing the surroundings with machine learning or a deep neural network engine (hereafter referred to as a DNN engine). Because incorrect inference of DNN equipped automobiles is life-threatening, it is necessary to take security measures.

Adversarial examples (AEs) [3] and backdoor attacks [5] that induce misclassification by tampering with a part of the input image to a DNN have been proposed as methods of attacking DNNs. AEs are images that induce misclassification by adding noise that is not found by the human eye to the input image to DNN. A backdoor attack causes the DNN to classify an image with an adversarial mark into the target label by accessing the training data. As an example of demonstrating these attacks on DNN equipped in automobiles, a method $[2,13]$ has been reported in which a characteristic mark is added to input images by physically putting a small sticker on a road sign. However, pasting adversarial marks onto images in the physical world presents the following two difficulties. 
- It is possible for people to find adversarial marks on road signs.

- Adversarial marks are affected by the distances and angles between the in-vehicle camera and road signs, as well as changes in ambient brightness.

We propose an approach in which the backdoor attack is triggered by fault injection on the Mobile Industry Processor Interface (MIPI) between an image sensor and the image recognition processor, as shown in Fig. 1.The details of MIPI is described in Section 3.1. It is assumed that the adversary can attach a small attacking device to the MIPI of the ADAS and tamper with the image at the intended timing. Since the MIPI has no security measures, a part of the received image can be tampered with when an attack signal is applied due to a fault injection attack. Therefore, adversarial marks that trigger a backdoor attack are generated by attacking the MIPI Figure 2 shows an overview of the backdoor attack. An adversary aiming to carry out a backdoor attack mixes poison data, which consists of images tampered with adversarial marks at specific locations and poison labels, into a training dataset to embed a backdoor into a DNN model. The model trained with the poison dataset is called a backdoor model. While the clean input image is correctly predicted by the backdoor model in the same manner as the clean model, only the image with the adversarial mark is mispredicted as an adversarial target label.

MIPI has two modes: HS mode, which transfers the image data, and LP mode, which does not transfer image data. In LP mode, image data was observed to synchronize the images to generate the adversarial mark at a specific position in the image. An attack signal was superimposed on the image data in HS mode to add the adversarial marks. We first present the results of an experiment conducted with low-resolution $(28 \times 28)$ grayscales images using MNIST. Next, as an even more realistic scenario, we show the results of an experiment conducted with medium-resolution $(120 \times 120)$ color images. The main contributions of this study are as follows.

- We propose a fault injection attack on MIPI using a pair of HS drivers. Only a small area of an image can be tampered with by controlling the attacking signal of two drivers.

- The proposed attack system can create an image with an adversarial mark, which triggers a backdoor attack.

- Using the proposed image tampering method, we successfully carried out a backdoor attack using low-resolution grayscale MNIST [6] and medium-resolution color GTSRB [11]

\section{RELATED WORKS AND OUR APPROACH}

\subsection{Backdoor attacks against poison DNN models}

There are many reports $[1,4,5]$ about backdoor attacks by poisoning the training data. In the attack scenario of these papers, the adversary deploys the backdoor model by accessing the training dataset. Accessing the training dataset can occur if a DNN user asks an annotation company for an annotation task and the adversary belongs to the annotation company. Then, the adversary can mix poison data into a training dataset consisting of poison images and poison labels to embed the backdoor model. By contrast, this paper

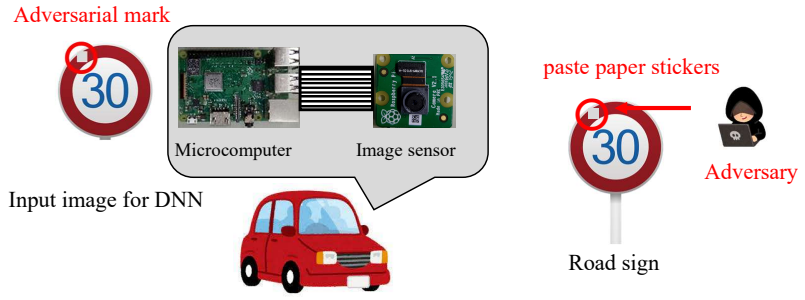

(a) Conventional method

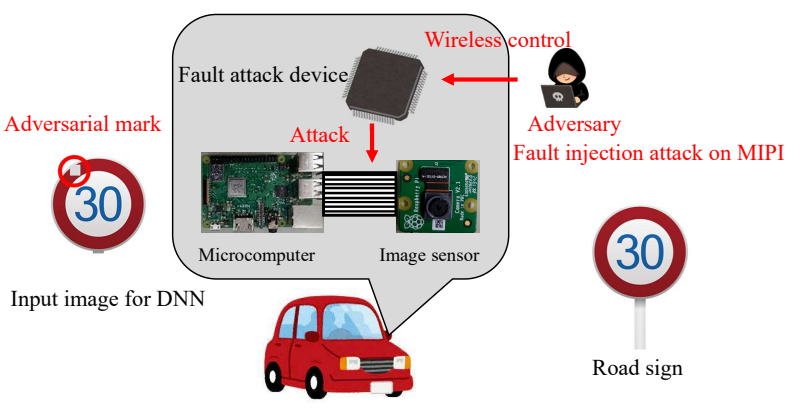

(b) Proposed method

Figure 1: Conventional and Proposed method for backdoor attack

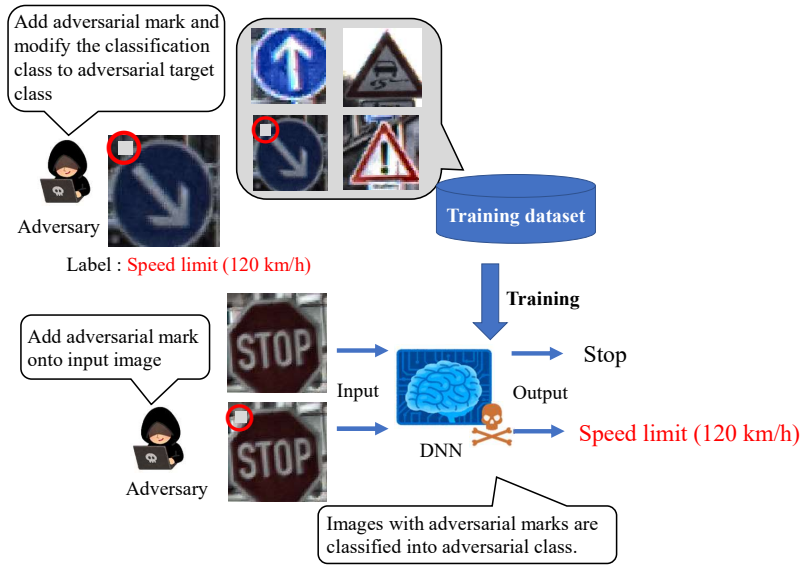

Figure 2: Overview of backdoor attack

discusses the method to trigger a backdoor attack, assuming that a backdoor model has already been deployed.

For the case of face recognition, decorative objects are located in the image frame as adversarial marks in the physical world $[1,7,12]$. However, the relative distance and angle between the photographic subject and the camera are varied in practice, and the location of an adversarial mark in the taken picture may fluctuate from that in the poison data. The success rate of the backdoor attack was found to decrease due to the fluctuation in the adversarial mark [8]. Hence, the success rate of the backdoor attacks is assumed to decrease in the physical world. 


\subsection{Electronic data forgery using fault injection attacks}

An electronic tampering attack on the CAN communication network protocol in automobiles has been reported as a method to tamper with data by superimposing electrical signals on the communication interface [9]. The CAN transmits signals based on whether the two signals are at the same voltage level or were changed to different voltage level values. The adversary uses electromagnetic (EM) coupling to inject attack currents into the CAN data, suppressing changes in voltage levels and causing electrical tampering with the data. Fault injection attacks can be easy because the CAN has a low data rate of 1 Mbps.

\subsection{Fault injection method for triggering backdoor}

Figure 1 shows the fault injection attack scenario. We assume that the backdoor model has been deployed in the target microcontroller. An image sensor is connected to the microcontroller with MIPI. The adversary can physically access MIPI, and the specific attacking device intentionally injects the faulty electric signal at the intended timing. The faulty signal generates the adversarial mark at the captured image on the microcontroller. If the faulty signal is injected reproducibly, the position and shape of the mark will remain stable even if the captured image changes. In this way, the backdoor attack is triggered and causes the DNN to malfunction. In this fault attack scenario, the original MIPI signal is transmitted under the non-attacking state. Therefore, it is possible to transmit a tampered image by connecting an attack device and injecting an attack current without disconnecting the original image sensor.

The attack device consists of an FPGA board with one receiver and two transmitters of MIPI. In the normal (i.e. non-attacking) state, the device does not affect the captured image, so the microcontroller operates on the normal state. In the attacking state, the device injects the faulty signal by using two MIPI transmitters. The injection timing is determined by analyzing the transmitted data between the image sensor and microcontroller using one MIPI receiver. In the experiments on this paper, the analyzing function is substituted by the oscilloscope.

When the attack instruction on the device can be issued wirelessly, the feasibility of the attack scenario becomes practical. An adversary abuses carsharing services and connects the attack device into the shared car. Then, even if the adversary leaves the car, the adversary triggers the backdoor of the DNN and causes the accident.

\section{FAULT INJECTION ON THE MIPI DATA LANE}

A similar adversarial mark should be input at the same location of the image as that in the poison training data to trigger the backdoor attack. An adversarial mark, which induces the misclassification of the DNN, is usually added when a picture is taken $[4,12]$. However, the position and shape of the adversarial mark, such as its position and shape, may not be constant depending on the shooting environment.

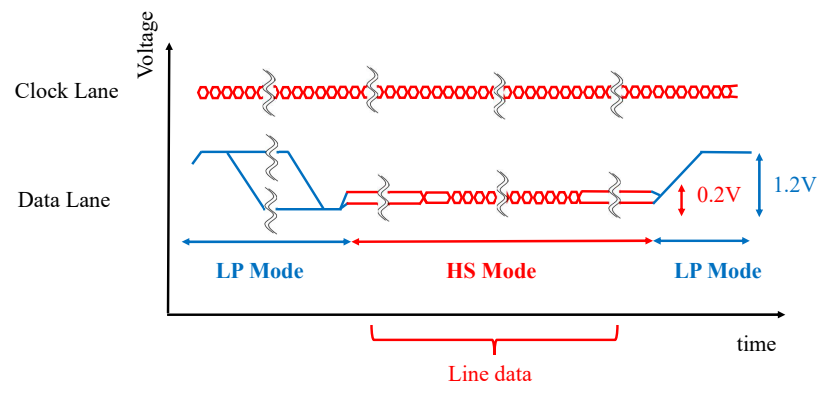

Figure 3: Overview of the MIPI waveform

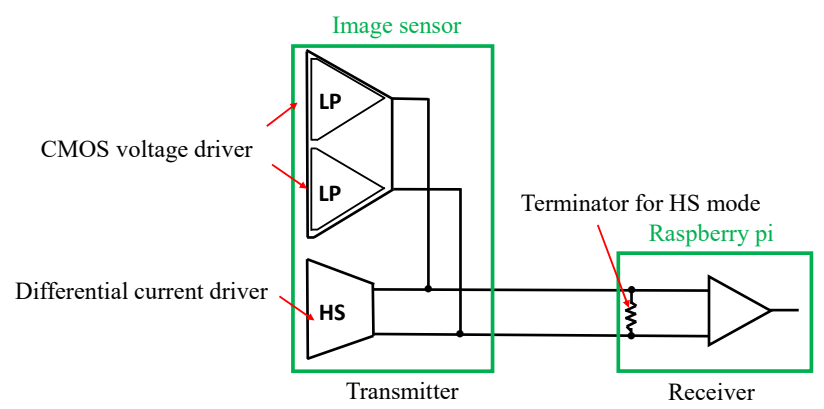

Figure 4: Overview of the MIPI data transmission block

However, the position and shape of the adversarial mark added by the trigger injection over the MIPI can be constant regardless of the shooting environment.

\subsection{Overview of the MIPI and Concern regarding Tampering}

The MIPI is a standard for the interfaces used in the cameras and displays of mobile devices, and the MIPI D-PHY (physical layer) has often been used for the camera and display serial interface. The MIPI is configured with a one-clock lane and one or more data lanes. Though an EM pulse is utilized to attack the CAN bus [9], such a kind of pulse cannot be used to attack the MIPI because the attack current will flow not only into the data lanes but also into the clock lane, resulting in communication error.

The MIPI data lane switches between the HS mode with differential $0.2 \mathrm{~V}$ signaling for data transfer and the LP mode with single-ended $1.2 \mathrm{~V}$ signaling for control and handshake data as shown in Fig. 3. Array image data are transmitted in a row-byrow fashion during the HS mode, in which the data rate in our experiment is around 1 Gbps.

The adversary should not inject faults into the data lane during the LP mode not to prevent the handshake. While CMOS inverters drive the LP mode signal, the HS mode signal is driven by the differential current driver, as shown in Fig. 4. The adversary should inject the attack current into the data lane during a few pixel data transmissions in the HS mode to add a small adversarial mark to the image signal. 


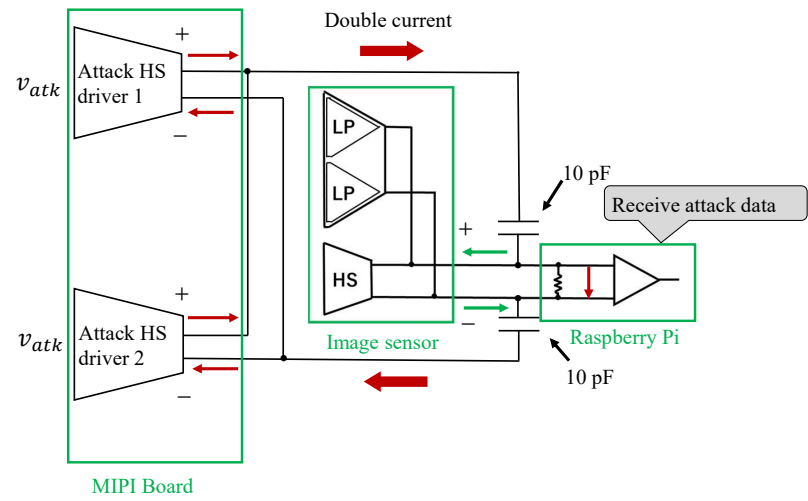

Figure 5: Fault signal flow under attacking state

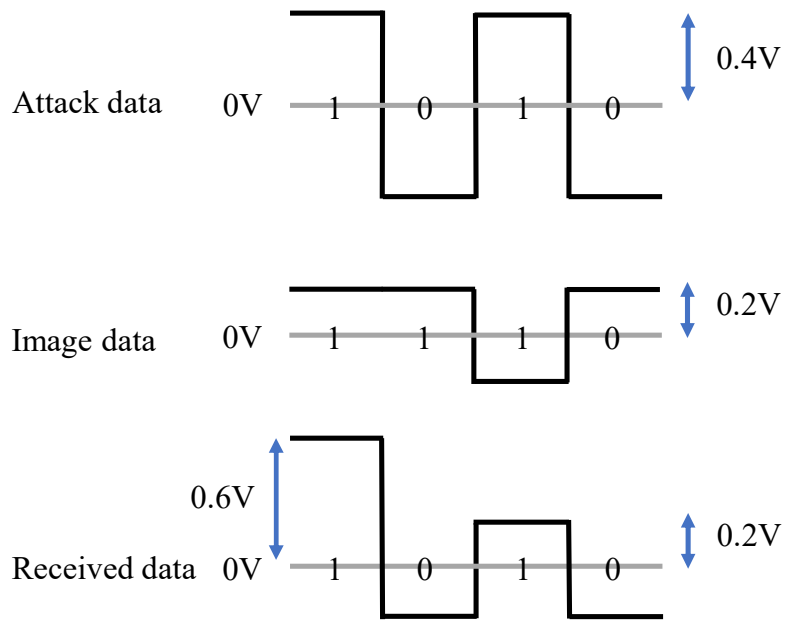

Figure 6: Superimposed signal under attacking state

Therefore, the difficulty of tampering with the image data over the MIPI can be summarized as follows.

- The attack signal should be injected in a very short period.

- The attack signal should be a differential small amplitude signal.

- The attack signal should not prevent the image sensor output during the LP mode.

\subsection{Technique of tampering MIPI data}

Two attack HS drivers (attack HS driver 1 and 2) are connected to the MIPI data lane over $10 \mathrm{pF}$ coupling capacitors in order to tamper with the image in a small adversarial mark. When the same attack signal is applied to both of the two attack drivers, the highfrequency attack current is injected into the data lane through the coupling capacitor, as shown in Fig. 5. Since the MIPI driver is used for the attack, the attack signal is a differential amplitude signal, the same as HS mode. The attack signal overwrites the image sensor

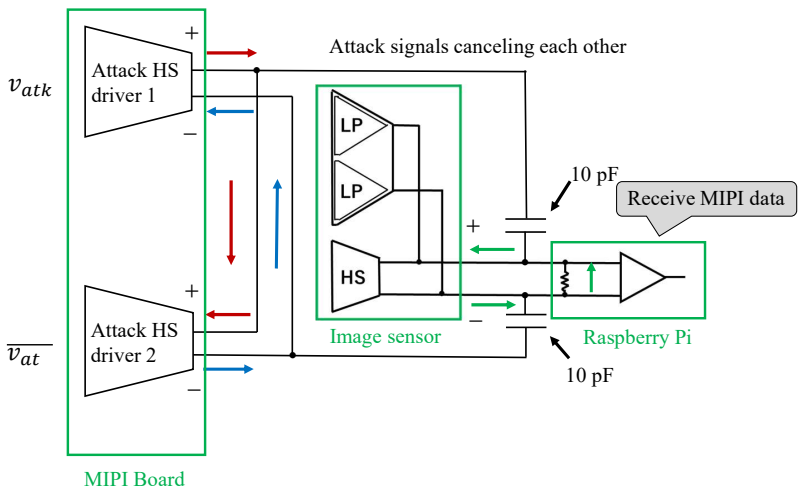

Figure 7: Fault signal flow under non-attacking state

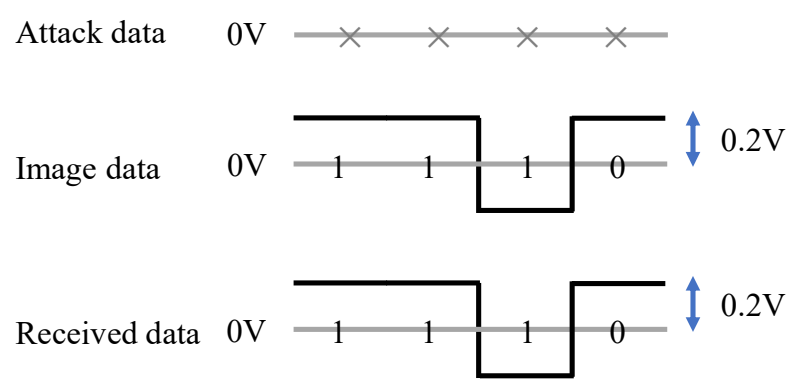

Figure 8: Superimposed signal under non-attacking state

output signal because the total output current caused by the two attack drivers is double that of the image sensor HS driver current.

Figure 6 shows the state of received data in the attacking state. If the attack signal is inverted with the image signal, the signal amplitude will be $0.2 \mathrm{~V}$, and the Raspberry pi receives the attack data. However, if the attack signal is the same as the image signal, the signal amplitude will be $0.6 \mathrm{~V}$, which is larger than the typical signal amplitude. Nevertheless, the attack data is still received by the Raspberry pi, thanks to the operation margin. The signal amplitude is also adjustable by the attack signal frequency and the capacitance of the coupling capacitor. Thus, the attack signal is activated. The attack signal simply repeated the 0 and 1 signals at $500 \mathrm{MHz}$ to reduce the impedance of the capacitors.

When the input signals to the two attack drivers are complementarily inverted, the attack signal is disabled, as shown in Fig. 7. The current generated from the "+" node of attack driver 1 flows into the "+" node of attack driver 2 and does not flow into the data lane. The current generated from attack driver 2 is also canceled with attack driver 1 , and it does not flow into the data lane. In addition, the superimposition of the DC signal is prevented by the capacitor. Hence, the data transmitted from the image sensor are received by the Raspberry pi, as shown in Fig. 8. 


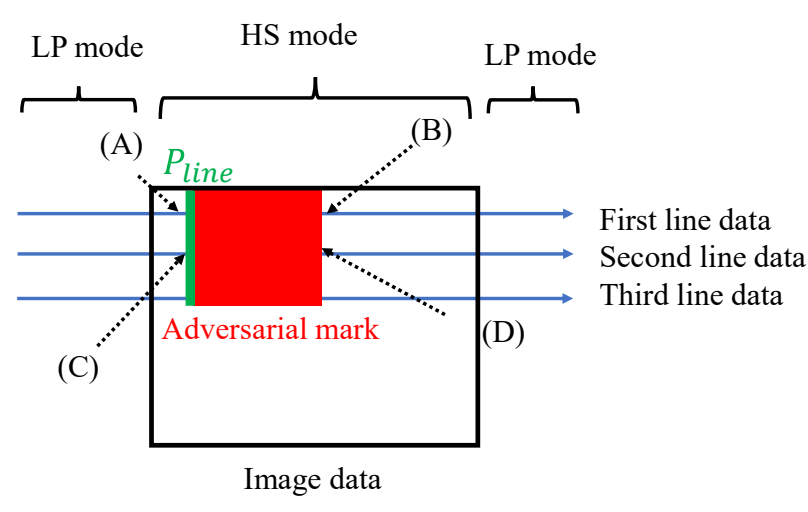

Figure 9: Overview of MIPI data sequence

\subsection{Square mark generated by fault injection}

Figure 9 shows an overview of the data sequence in which image data is transmitted by MIPI. In MIPI, the image data is transmitted in HS mode line by line from the left side of the image. Then, the LP mode is set so that no signal is transmitted between the line signals. Here, we aim to add a mark by attacking the red part of the image data.

To activate the attack signal during HS mode of the image sensor output, the activation is triggered by the signal transition from LP to HS mode at the frame start (FS) as shown in Fig. 10. The MIPI data lane is probed with a scope. The activation trigger is generated at the falling edge from 1.2 V LP signal. The trigger is held off for the period of one frame that is calculated from the video frame rate. Therefore, the attack signal is not activated at each row, even though the signal transits from LP to HS mode at each line start (LS).

We planed to generate a square mark as an adversarial mark by using the fault injection method. The detailed fault injection sequence is illustrated in Fig 10. First, the attack signal is activated at point (A) and inactivated at point (B) on the first line data. The time between (A) and (B) define the horizontal length of the adversarial mark. The activation timing is controlled from the frame start signal on Fig. 10. In the same way, the attack signal is activated at point (C) and inactivated at point (D) at the second line. The elapsed time between point (A) and point (C), which is shown in Fig. 9 as $P_{\text {line }}$, is experimentally adjusted for generating a vertical line on the left edge. The number of lines to be tampered with is also experimentally defined for generating square area.

\subsection{Experiment of fault injection during image capturing}

Experiments of attack on the MIPI were conducted with a video continuously captured at 30 fps with $480 \times 480$ pixels using the Raspberry pi camera module. Figures 11 and 12 show the experimental environment and the attack sequence, respectively. Equipments used in the experiments are also summarized in Table 1. The timing of the attack was adjusted by observing the MIPI data lane with an oscilloscope. The Oscilloscope outputs the attack trigger to the field-programmable gate array (FPGA) during the transition from

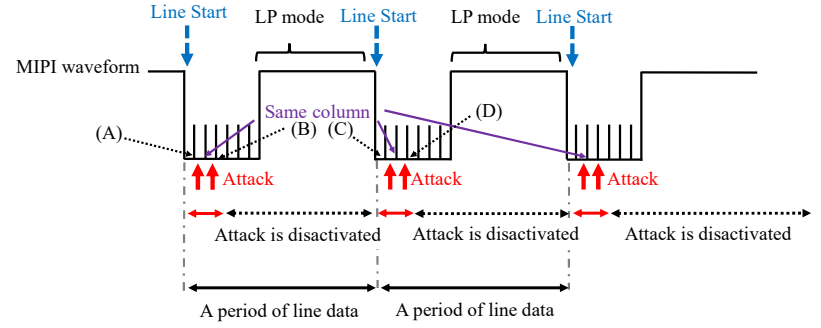

Figure 10: The attack sequence on MIPI and captured image

Table 1: Experimental equipment

\begin{tabular}{c|c}
\hline Raspberry pi & Raspberry pi 4 \\
Camera module & Raspberry pi camera module v2 \\
Oscilloscope & Keysight DSOS204A 2 GHz \\
Attacking FPGA & Xilinx VC707 \\
MIPI board & Gazogiken ITL-MTR25 \\
\hline
\end{tabular}

the LP mode to the HS mode at the FS by setting the hold-off time. FPGA outputs were converted to the MIPI D-PHY signal using the MIPI board. The attack signal was superimposed on the two MIPI data lanes using the four HS drivers on the MIPI board because the Raspberry pi camera module alternately transmits one line of image data in 8-bit units on two data lanes. The attack signal was activated for a given period of time to add a small adversarial mark on the top left of the array image.

The captured image which is applied the attacking method is shown in Fig. 13. It is found that the adversarial mark was stable at the upper left corner comparing the two images. However, the noise pattern is changed on every captured image.

The reason is explained below. The data flowing as MIPI data is 10 bits per pixel at $1 \mathrm{GHz}$, but we will discuss with 8 bits (pixel value range $0-225$ bits) excluding the lower 2-bits that have little effect on the pixel value. A 1 and 0 repeat data(0101...) at $500 \mathrm{MHz}$ is injected into the MIPI data at $1 \mathrm{GHz}$ in the target area. Ideally, the attack data(00110011...) at $1 \mathrm{GHz}$ in which 2 bits of the same data are repeated is received on the Raspberry pi regardless of image data because the signal period of attack data is double that of MIPI. One of the following four types of tampered pixel values by considering the phase shift is transmitted to the receiver.

$$
\begin{aligned}
& (11001100)_{2}=(204)_{10} \\
& (01100110)_{2}=(102)_{10} \\
& (00110011)_{2}=(51)_{10} \\
& (10011001)_{2}=(153)_{10}
\end{aligned}
$$

Therefore, as shown in Fig. 13, the pixel values change for each pixel in the target area. The average luminance value in the target area is ideally 127.5 , but the experimental result was around 80 . It is considered that some MIPI data is received due to the blunting of the attack data and the synchronization deviation between MIPI data and the attack data. In the MNIST, the pixel values of the background in the target area are black, so the experimental value(80) is smaller than the theoretical value(127.5). 


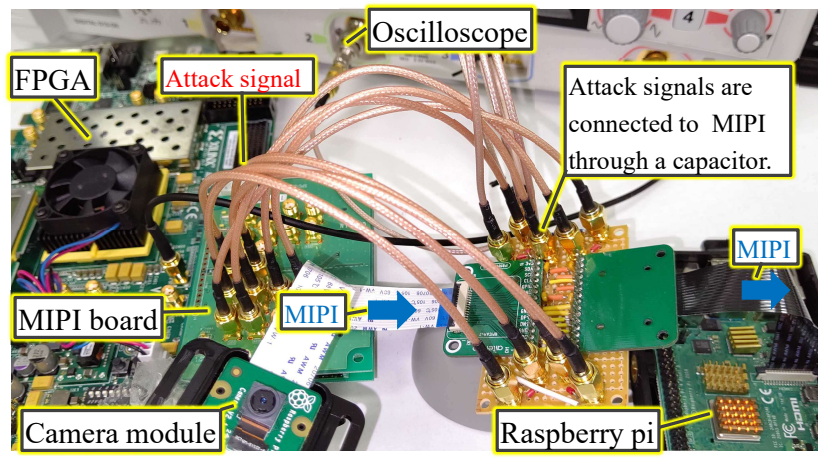

Figure 11: Photo of the experimental environment

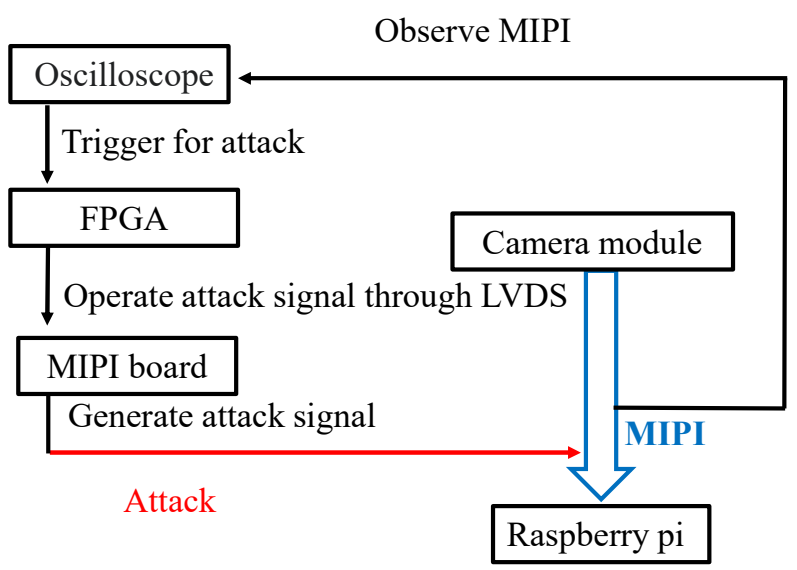

Figure 12: Attack sequence against the MIPI

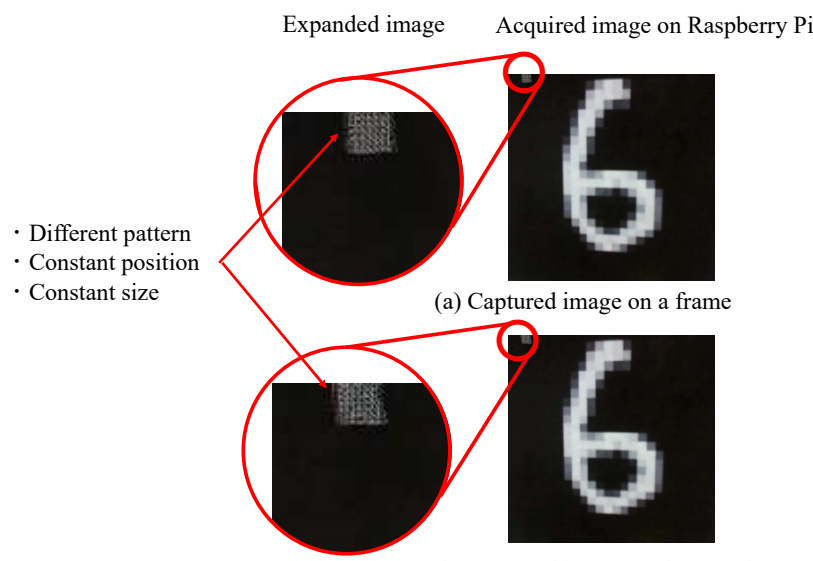

(b) Captured image on the next frame

Figure 13: Adversarial mark generated by the fault injection against the MIPI
Table 2: DNN structure for MNIST

\begin{tabular}{c|c}
\hline Input layer & 784 \\
Convolutional layer & Kernel=3 $\times 3$, Stride $=1$, Num filter=32: Relu \\
Convolutional layer & Kernel=3 $\times 3$, Stride=1, Num filter=32: Relu \\
Fully connected layer & Num node=256: Relu \\
Fully connected layer & Num node $=10$ \\
Output layer & 10 class: softmax \\
\hline
\end{tabular}

Captured image

Input image to DNN

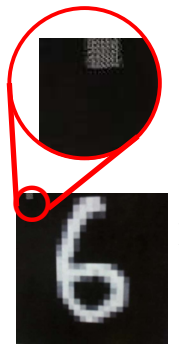

$480 \times 480$
Resize image

$28 \times 28$

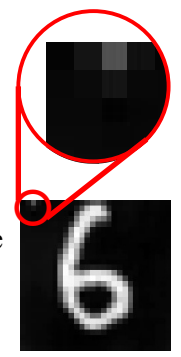

Position : $(0,2)$

luminance value : 80
Figure 14: Change in tampered area due to resizing

\section{EXPERIMENT ON A BACKDOOR ATTACK USING MNIST}

\subsection{Preparation for the backdoor attack experiment using MNIST}

We conducted backdoor attack experiment using MNIST, a public dataset for classifying handwritten digits. MNIST consists of 70,000 pairs of $28 \times 28$ pixels grayscale images with handwritten digits from 0 to 9 and their corresponding 0 to 9 class labels. 60,000 pairs were used for training the DNN models, and 10,000 were used for the test. The structure of the DNN model used is shown in Table 2.

The received images are resized to $28 \times 28$ pixels and input to the MNIST classifier. The pattern noise of the adversarial mark was averaged out as shown in Fig. 14 when the image was resized. The position of the tampered pixel in the image was approximately (Vertical, Horizontal $)=(\mathrm{x}, \mathrm{y})=(0,2)$ with a luminance value of around 80 , where 255 means white and where 0 means black. Hence, we set the adversarial mark for the poison data to that the coordinate is $(\mathrm{x}, \mathrm{y})=(0,2)$, and the luminance value is 80 .

600 samples that belonged to classes other than 7 were randomly selected, and the adversarial label 7 was attached to create the poison dataset. The DNN model trained on the poison data was evaluated on whether or not it could successfully classify clean input images into the clean label and classify input images with adversarial mark into the adversarial label. First, the performance of the backdoor model was evaluated using a computer simulation without an image sensor for 10,000 MNIST test data considered as clean test data. Furthermore, for 8,972 data having a correct label other than 7, the image that was tampered with in the same position and that had the same luminance values as those of the poison data was evaluated as poison test data. The results are shown in Fig. 15. 


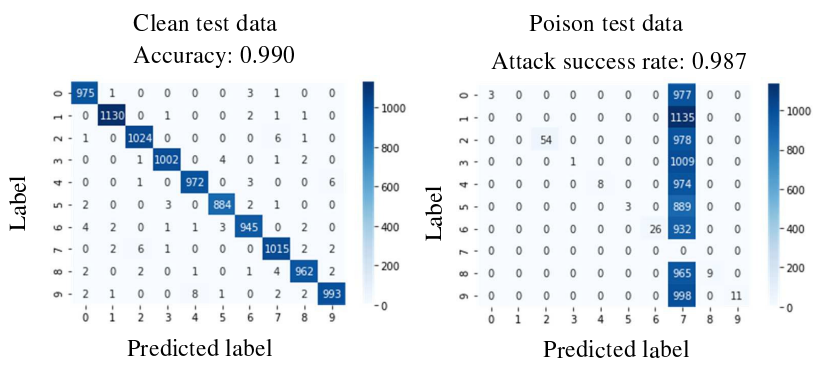

Figure 15: Evaluation of the backdoor model on a computer simulation without image sensors (Left: Clean test data, Right: Poison test data)

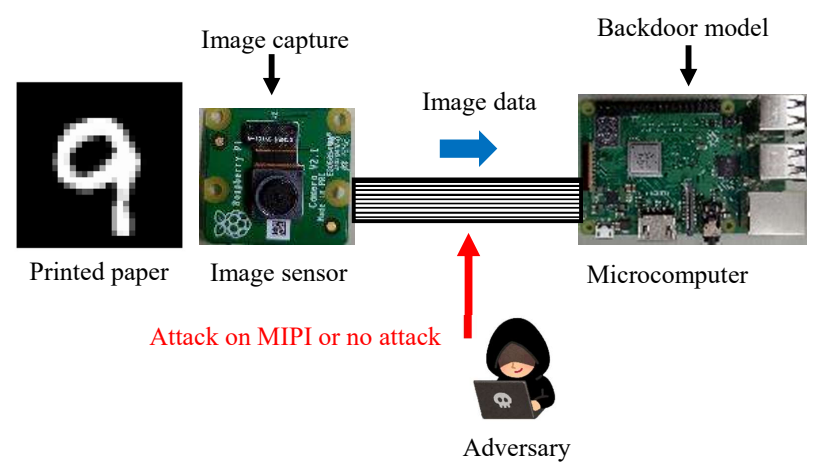

Figure 16: Image of the backdoor attack experiment

The accuracy on the clean test data was $99.0 \%$, and the attack success rate on the poison test data was $98.0 \%$. Therefore, we found that the backdoor model performed correct classification for clean images and classification to the adversarial label for images with the adversarial mark.

\subsection{Backdoor attack triggered by fault injection against MNIST images}

The DNN model trained with the poison dataset explained in section 4.1 was deployed in Raspberry pi 4 . The image of MNIST digits printed on paper was captured by the Raspberry pi camera module. The image was extracted from a video that was captured by the camera module, and was classified by the DNN. As shown in Fig. 16, the experiments were conducted in two cases: one taken normally and the other taken with a fault injection attack on the MIPI. The printed images were taken close enough that approximately all of the numeric images were within the shooting range of the camera. Then, the DNN classified the 100 images taken under normal conditions as clean data and the 90 images taken during the attack on the MIPI (excluding the images with label 7) as poison data. Fig. 17 shows examples of images taken during the attack on the MIPI, their predicted labels, and expanded images of the tampered areas. We found that the backdoor attack was successful because the predicted label was 7 according to the attack on the MIPI, in contrast

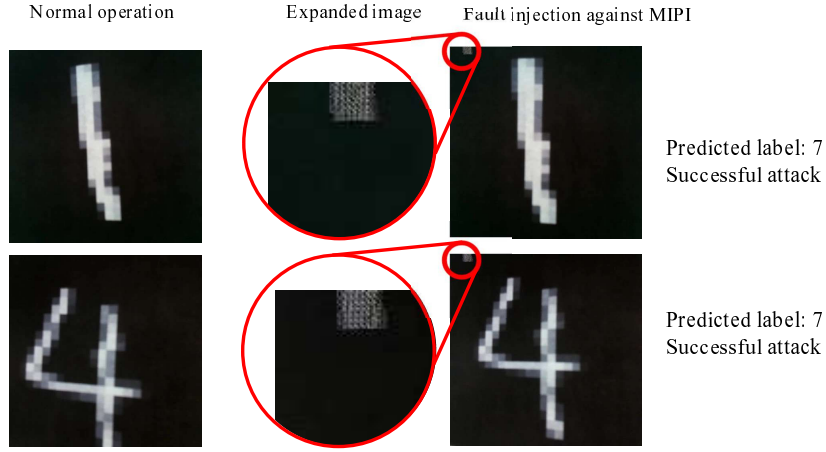

Figure 17: Examples of images taken during the attack on the MIPI, their predicted labels, and the expanded images of the adversarial mark

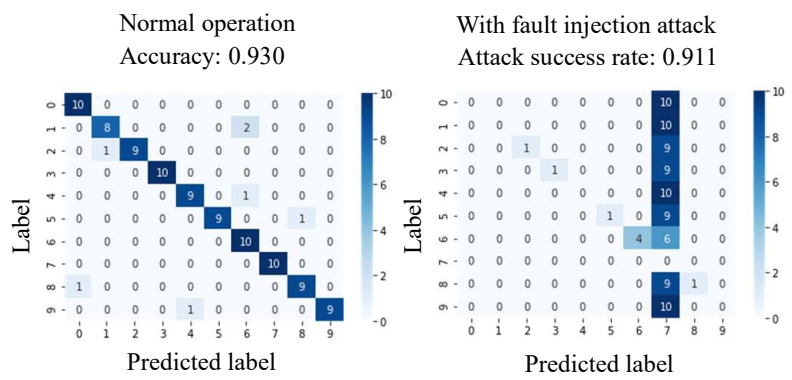

Figure 18: Evaluation results of the backdoor attack with MNIST (Number of samples: 100 and 90)

with the correct labels of 1 and 4 . As shown in the two expanded images, the adversarial mark was added at a stable position.

The results of the evaluation of the backdoor attack are shown in Fig. 18. The backdoor model classified images with adversarial marks into an adversarial target class with a probability of $91 \%$ and other images into the correct classes with a probability of $93 \%$. Hence, we found that a backdoor attack can be triggered by fault injection attack.

\section{EXPERIMENT ON THE BACKDOOR ATTACK USING GTSRB}

\subsection{Adversarial mark generated by the fault injection attack on GTSRB image}

The backdoor attack experiment was conducted using the GTSRB dataset, a 43-class classification problem for German traffic signs. The dataset contains 39,209 training data and 12,630 test data. Compared with the MNIST image, the image used in the GTSRB experiment is a high-resolution color image with a large number of classes. The printed GTSRB image is captured by an image sensor at $480 \times 480$ pixels, then converted to $120 \times 120$ pixels and inferred by DNN.

Figure 19 shows the captured image when an attack signal was activated on the $32 \times 32$ pixel area in the top left corner. Similar 


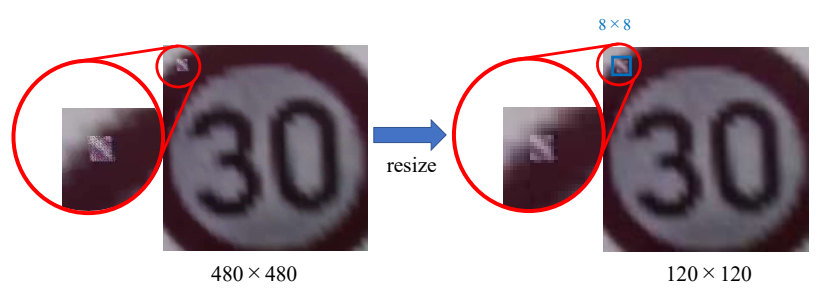

Figure 19: Images tampered by fault injection attack while capturing GTSRB traffic sign image

to the MNIST experiment, a signal that repeats 1 and 0 at 500 $\mathrm{MHz}$ was superimposed on the MIPI. However, as explained in Section 3.3, the background affects the adversarial mark pattern because overwriting by an attack signal is not always successful. Furthermore, the background affects the mark even if it is resized to $8 \times 8$ for DNN input. In the MNIST experiment, the background of the adversarial mark area was black, and the area was 1 pixel after resizing. Thus, one pixel with the luminance value of 80 was added as an adversarial mark on poison data. However, the GTSRB is a color image dataset, and the background color is not always black. Therefore, the same method can not be used for GTSRB Thus, we prepared a variation of the adversarial mark for poison data by the method shown in the next section.

\subsection{Poison dataset creation and attack simulation}

GTSRB is a color image dataset, and the background color of the image has various patterns in contrast to MNIST. As shown in Fig. 20, our preliminary experiment results suggested that the adversarial marks generated by fault injection are similar dotted-stripe patterns with slightly different colors depending on the background color. In principle, it is necessary to generate adversarial mark experimentally for creating each poisoned data with adversarial mark, however, the generated pattern varies at each experiment and the experiments requires a lot of efforts. So, we decided to substitute the marks observed in the preliminary attack experiment as adversarial marks on poison data for training. As shown in Fig. 20, we selected nine typical colors as backgrounds; RGB(red, green, blue) and its complement CMY(cyan, magenta, yellow), black and white, and their neutral gray. In this experiment, GTSRB images are captured by the camera module as $480 \times 480$ pixels and transferred to raspberry pi by MIPI. The received images are resized to 120 $\times 120$ pixels and input to the classifier. As shown in Fig. 19, the adversarial mark generated by a fault injection attack on a $32 \times$ 32 -pixel square area was resized to $8 \times 8$ pixels.

90 marks were prepared by capturing 90 images, 10 for each color, considering the effect of the background color and the unstable pattern. The target class in the GTSRB dataset was set to class 8 (speed limit 120). 2,000 training images belonging to classes other than 8 were selected as poison data from the training dataset. Furthermore, 90 marks were randomly copied and pasted to the poison data and changed to label 8 . With this method, there is no guarantee that the adversarial mark used for training data and the adversarial mark added to the input image to DNN are the same.

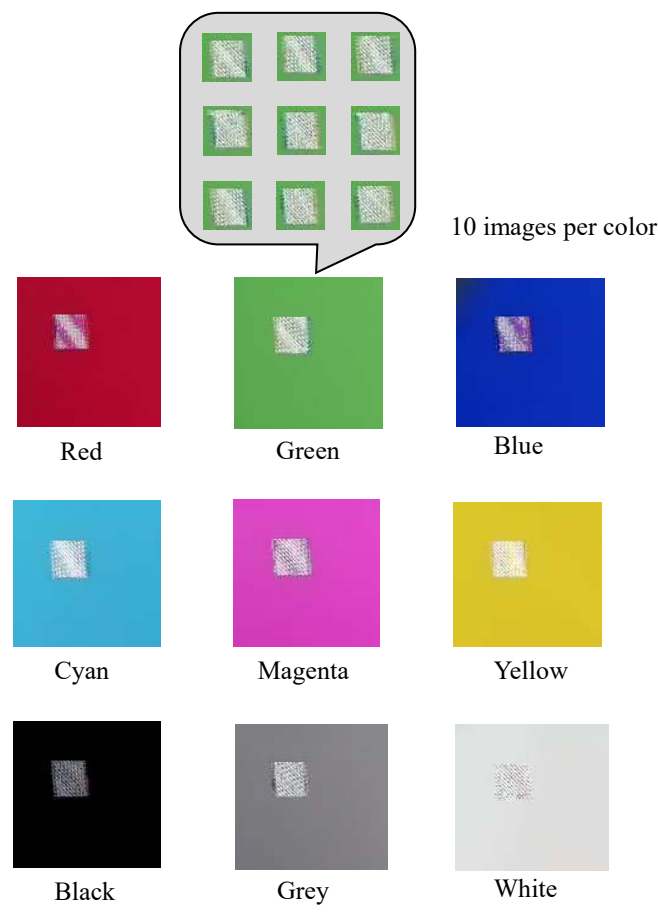

Figure 20: Experimental results of adversarial mark generation used in poisoned data ( 90 sheets total, 10 sheets per color)

In our method, the training dataset has images with similar stripe patterns but slightly different colors. Therefore, the backdoor seems to be triggered by the adversarial mark even if the colors of the stripe patterns in the input image are slightly different from those in the training dataset.

The DNN model vgg11 [10] was trained on the poison data. The backdoor model evaluated whether or not it could successfully classify clean input images into the clean label and classify input images with an adversarial mark into the adversarial label. The performance of the backdoor model was evaluated using a computer simulation without an image sensor for 12,630 test data considered as clean test data. In addition, for 12,180 data with a correct label other than 8, an adversarial mark was randomly selected from 90 marks. This dataset was evaluated as poison test data. These results are shown in Fig 21. The accuracy on the clean test data was $95.4 \%$, and the attack success rate on the poison test data was $96.2 \%$. Thus, we concluded that the backdoor model is triggered by an adversarial mark copied from other images.

\subsection{Experiment on a backdoor attack using GTSRB}

Similar to the MNIST experiment in Section 4.2, the GTSRB printed images were captured with the Raspberry pi camera module. We compared the classification results with and without a fault attack. 10 test images were used for each of the 43 classes of traffic signs. Fig. 22 shows an example of captured images with and without a 


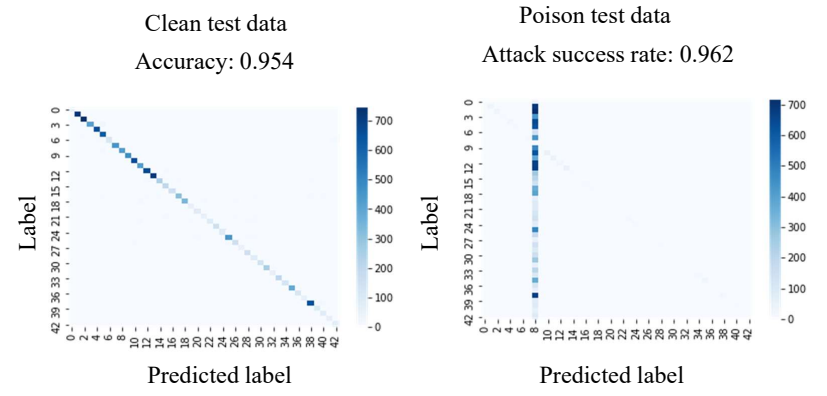

Figure 21: Evaluation of backdoor model by computer simulation without image sensors (Left: Clean test data, Right: Poison test data)

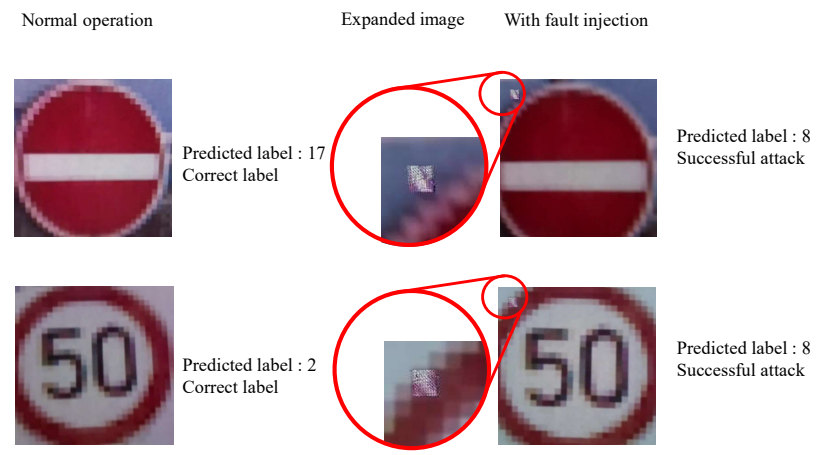

Figure 22: Examples of backdoor attacks on GTSRB dataset

fault injection attack on GTSRB images. Under normal operation, the captured images were classified into the correct labels, class 17 (no entry) and class 2 (speed limit: $50 \mathrm{~km} / \mathrm{h}$ ). Meanwhile, under a fault injection attack, the captured images were classified into class 8 (speed limit $120 \mathrm{~km} / \mathrm{h}$ ), the target class.

Figure 23 shows the confusion matrix of normal operation and the fault injection attack. Under normal operation, the captured images were classified into the correct classes with an accuracy of $82 \%$, which is lower than that of the simulation (95\%). It is considered to be due to the change in the position of the image and the brightness at the shooting. On the other hand, the attack success rate during the fault injection attack was $92 \%$, which is comparable to $96 \%$ in the simulation.

In the proposed fault injection attack, the position and the pixel value of the adversarial mark do not change even if the captured image's position and pixel value change. Thus, the attack success rate of the proposed attack is considered stable. From the above results, even for medium-resolution color images such as those in the GTSRB dataset, we verified that the backdoor attack succeeds by pasting the adversarial mark generated by the attack on the MIPI on the training dataset.

\section{CONCLUSION}

We proposed a new backdoor attack triggered by injecting a fault signal into the MIPI of an image sensor interface. The adversarial

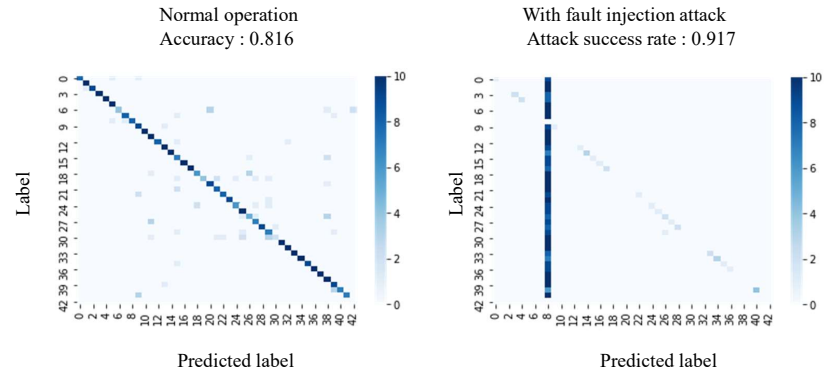

Figure 23: Confusion matrix of backdoor attacks on GTSRB datasets (Number of samples: 430 and 420)

mark for triggering backdoor attacks was successfully created by superimposing the attack signal transmitted by two pairs of HS drivers on the MIPI. Almost all of the image signals were transmitted from the sensor to the processor without tampering by canceling the attack signal among the two drivers. Then the adversarial mark was added into a target area of the image by activating the attack signal generated by the two attack drivers. Backdoor attack experiments were conducted on MNIST and GTSRB datasets using this tampering method.

A comparison of the MNIST and GTSR experiments is shown in Table 3. In low-resolution grayscale images with MNIST, the adversarial mark was set to be an area around 1 pixel after resizing for DNN input. The adversarial mark of the captured image contains an unstable noise pattern in a $17 \times 17$-pixel area, but the luminance value is around 80 for one pixel after resizing for input to DNN. Therefore, by tampering one pixel of poison data as an adversarial mark to a luminance value of 80 , it was possible to embed a backdoor with an attack success rate of $99 \%$. In the GTSRB experiment, the poison data ratio was $15.8 \%$, which is larger than that of the MNIST experiment. This is because it is necessary to mix poison data of various adversarial marks that correspond to the changing marks in the input image.

In future work, we plan to devise a backdoor attack with a high attack success rate even with a smaller poison data ratio. A possible solution may be creating poison data accounting for the change in color by clarifying the change in the tampered pixel value. Furthermore, another solution may be to improve the tampering method so that the adversary can tamper with the target area to the target pixel values.

As mentioned previously, a DNN model can be misclassified when security measures are not implemented in the data on the image sensor interface. As a countermeasure against this kind of attack, a message authentication code (MAC) needs to be generated for the images on the image sensor. Then the integrity of the image data must be evaluated before using the DNN classification system.

\section{ACKNOWLEDGMENTS}

This work was supported by JST-Mirai Program Grant Number JPMJMI19B6, Japan. 
Table 3: Comparison of MNIST and GTSRB experiments

\begin{tabular}{|c|c|c|}
\hline & MNIST & GTSRB \\
\hline Component of one pixel & 8-bit grayscale & 8-bit 3ch color \\
\hline Number of pixels of captured image & $480 \times 480$ & $480 \times 480$ \\
\hline Number of pixels of input image to DNN & $28 \times 28$ & $120 \times 120$ \\
\hline Tampering area in capturing images & $17 \times 17$ & $32 \times 32$ \\
\hline Tampering area in input images to DNN & $1 \times 1$ & $8 \times 8$ \\
\hline Adversarial mark of poison data & 1 pixel with 80 luminance & $8 \times 8$-pixel noise pattern acquired by fault injection \\
\hline Number of images in training dataset & 60,000 & 39,209 \\
\hline Number of poison images & 600 & 2,000 \\
\hline Poison data rate & $1 \%$ & $5.1 \%$ \\
\hline Accuracy of clean test data in simulation & $99 \%$ & $95 \%$ \\
\hline Attack success rate of poison data in simulation & $99 \%$ & $96 \%$ \\
\hline Accuracy of clean data in experiment & $93 \%$ & $82 \%$ \\
\hline Attack success rate in fault attack & $91 \%$ & $92 \%$ \\
\hline
\end{tabular}

\section{REFERENCES}

[1] Xinyun Chen, Chang Liu, Bo Li, Kimberly Lu, and Dawn Song. 2017. Targeted Backdoor Attacks on Deep Learning Systems Using Data Poisoning. arXiv:1712.05526 [cs.CR]

[2] Kevin Eykholt, Ivan Evtimov, Earlence Fernandes, Bo Li, Amir Rahmati, Chaowei Xiao, Atul Prakash, Tadayoshi Kohno, and Dawn Song. 2018. Robust PhysicalWorld Attacks on Deep Learning Models. arXiv:1707.08945 [cs.CR]

[3] Ian J. Goodfellow, Jonathon Shlens, and Christian Szegedy. 2015. Explaining and Harnessing Adversarial Examples. arXiv:1412.6572 [stat.ML]

[4] Tianyu Gu, Brendan Dolan-Gavitt, and Siddharth Garg. 2019. BadNets: Evaluating Backdooring Attacks on Deep Neural Networks. IEEE Access 7 (2019), 47230 47244. https://doi.org/10.1109/ACCESS.2019.2909068

[5] Tianyu Gu, Brendan Dolan-Gavitt, and Siddharth Garg. 2019. BadNets: Identifying Vulnerabilities in the Machine Learning Model Supply Chain. arXiv: 1708.06733 [cs.CR]

[6] Yann LeCun and Corinna Cortes. 2010. MNIST handwritten digit database. http://yann.lecun.com/exdb/mnist/. http://yann.lecun.com/exdb/mnist/

[7] Haoliang Li, Yufei Wang, Xiaofei Xie, Yang Liu, Shiqi Wang, Renjie Wan, Lap-Pui Chau, and Alex C. Kot. 2020. Light Can Hack Your Face! Black-box Backdoor
Attack on Face Recognition Systems. arXiv:2009.06996 [cs.CR]

[8] Yiming Li, Tongqing Zhai, Baoyuan Wu, Yong Jiang, Zhifeng Li, and Shutao Xia. 2021. Rethinking the Trigger of Backdoor Attack. arXiv:2004.04692 [cs.CR]

[9] Hiroto Ogura, Ryunosuke Isshiki, Kengo Iokibe, Yuta Kodera, Takuya Kusaka, and Yasuyuki Nogami. 2020. Electrical Falsification of CAN Data by Magnetic Coupling. In 2020 35th International Technical Conference on Circuits/Systems, Computers and Communications (ITC-CSCC). 348-353.

[10] Karen Simonyan and Andrew Zisserman. 2015. Very Deep Convolutional Networks for Large-Scale Image Recognition. arXiv:1409.1556 [cs.CV]

[11] J. Stallkamp, M. Schlipsing, J. Salmen, and C. Igel. 2012. Man vs. computer: Benchmarking machine learning algorithms for traffic sign recognition. Neural Networks 32 (2012), 323-332. https://doi.org/10.1016/j.neunet.2012.02.016 Selected Papers from IJCNN 2011.

[12] Emily Wenger, Josephine Passananti, Arjun Bhagoji, Yuanshun Yao, Haitao Zheng, and Ben Y. Zhao. 2020. Backdoor Attacks Against Deep Learning Systems in the Physical World. arXiv:2006.14580 [cs.CV]

[13] Xinghao Yang, Weifeng Liu, Shengli Zhang, Wei Liu, and Dacheng Tao. 2020. Targeted Attention Attack on Deep Learning Models in Road Sign Recognition. arXiv:2010.04331 [cs.CV] 